\title{
Thoracic spinal cord impingement by an arachnoid web at the level of a hemivertebra: case report
}

\author{
Molly E. Hubbard, MD, ${ }^{1}$ Matthew A. Hunt, MD, ${ }^{1}$ Kristen E. Jones, MD, ${ }^{1}$ and David W. Polly, MD² \\ Departments of ${ }^{1}$ Neurosurgery and ${ }^{2}$ Orthopaedic Surgery, University of Minnesota, Minneapolis, Minnesota
}

Congenital scoliosis due to a hemivertebra requires surgical stabilization prior to skeletal maturity if rapidly progressive curve growth occurs. Here the authors present the unique case of a man who, at the age of 12 years, had undergone Harrington rod placement for stabilization of progressive congenital scoliosis due to a T-11 hemivertebra and then, at the age of 53 years, presented with acutely progressive myelopathy due to spinal cord compression from an arachnoid web at T-11 despite a solid fusion mass at the prior surgical site. The patient underwent a posterior midline approach for resection of the T-11 pedicle at the level of the hemivertebra, intradural spinal cord detethering with resection of the arachnoid web at T-11, and T2-L2 instrumented fusion with deformity correction, leading to subsequent resolution of his acute myelopathic symptoms. In conclusion, arachnoid web formation superimposed on preexisting tension on the thoracic spinal cord from congenital scoliosis due to a T-11 hemivertebra caused acute myelopathy in an adult with a previously solid fusion mass from childhood. The resolution of acute myelopathy and halting of further progression occurred with pedicle resection, arachnoid web fenestration, and spinal deformity correction.

https://thejns.org/doi/abs/10.3171/2017.5.SPINE161243

KEY WORDS hemivertebra; arachnoid web; myelopathy; adult; thoracic; congenital scoliosis

$\mathrm{P}$ ATIENTS with a hemivertebra leading to scoliosis require fusion if imaging reveals progression of the scoliotic curve. In this case report, we describe a patient who, 41 years after his initial fusion, developed worsening symptoms of myelopathy below the level of a known hemivertebra. His fusion mass appeared stable, but he was found to have an arachnoid web at the level of the hemivertebra. We describe the surgical approach performed in an attempt to halt progression of his symptoms.

\section{Case Report}

History and Examination

A 53-year-old gentleman presented with acute progressive myelopathy in his lower extremities. His history included T6-L1 Harrington rod placement at 12 years of age in 1975 for progressive congenital scoliosis due to a T-11 hemivertebra. His current symptoms began with right leg numbness that progressed over 4 months to bilateral lowerextremity burning dysesthesias and gait instability, as well as difficulty with ambulation. On physical examination, he had patellar and Achilles hyperreflexia, ankle clonus, nondermatomal decreased sensation in his lower extremities, and an inability to perform tandem gait. Standing longcassette radiographs showed a left T-11 hemivertebra with $52^{\circ}$ right thoracolumbar scoliosis and bilateral Harrington rod instrumentation with hooks spanning T6-L1 (Fig. 1). Thoracolumbar MRI was nondiagnostic because of metallic artifact from the Harrington rods (Fig. 2). Computed tomography myelography demonstrated significant narrowing of the spinal canal at the T-11 level (Fig. 3). Close inspection of the myelogram revealed an intradural source of cord compression dorsal to the spinal cord at the T-11 level, suggestive of an arachnoid web. A dense posterolateral fusion mass was noted from T-3 to T-12. His previous fusion appeared to be solid without evidence of pseudarthrosis.

The patient was seen in both the orthopedic and neurosurgical clinics for preoperative planning and discussion of the surgical plan. Given his myelopathic changes and 

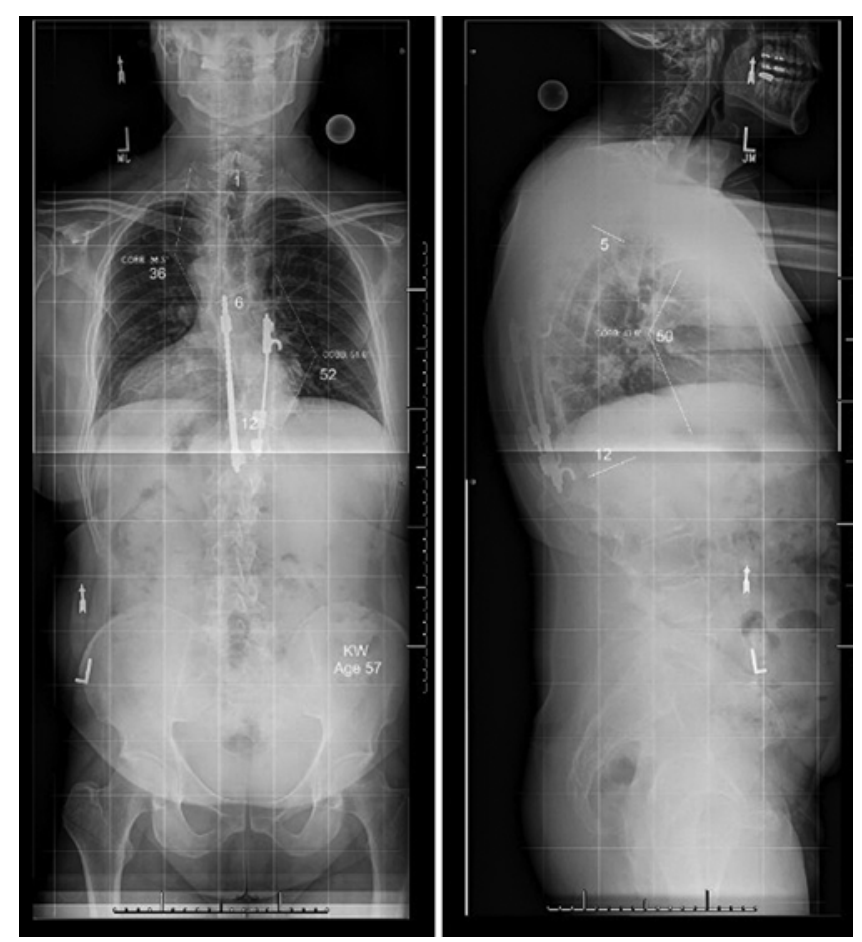

FIG. 1. Preoperative standing long-cassette radiographs showing Harrington rods from T-6 to L-1 and $52^{\circ}$ right thoracolumbar scoliosis.

progression of symptoms, as well as a solid-appearing fusion mass, the initial plan was to perform removal of the instrumentation and decompression of the tethered area.

\section{Operation}

Because of progressive myelopathy with intradural cord compression at $\mathrm{T}-11$, the patient was taken to the operating room for removal of the Harrington rods, T10-12 decompressive laminectomy, a transpedicular approach for pedicle resection at the level of the left T-11 hemivertebra, intradural exploration with arachnoid web excision, and T2-L2 posterior instrumented segmental fusion with pedicle screws, all with intraoperative neuromonitoring. The fusion included the high thoracic spine to stabilize across the curve at the T3-4 hemivertebra. Resection of the T-11 pedicle and the hemivertebra had been discussed with the patient preoperatively. During the surgical procedure, the pedicle was seen to be causing deformity and compression of the cord; thus, resection was necessary to obtain adequate decompression. After this resection, decreased extradural tension on the thecal sac was noted intraoperatively. Unfortunately, this did destabilize the area, necessitating a redo arthrodesis. Intradural exploration at T-11 revealed a thickened band of arachnoid tissue over the dorsal aspect of the cord (Fig. 4). With the use of a microscope, the band was sharply resected. Resection of the arachnoid web resulted in further normalization of the lateral spinal cord translation. Intraoperative neuromonitoring with somatosensory evoked potentials and motor evoked potentials of the lower extremities remained stable following the hemivertebra and arachnoid web resection.

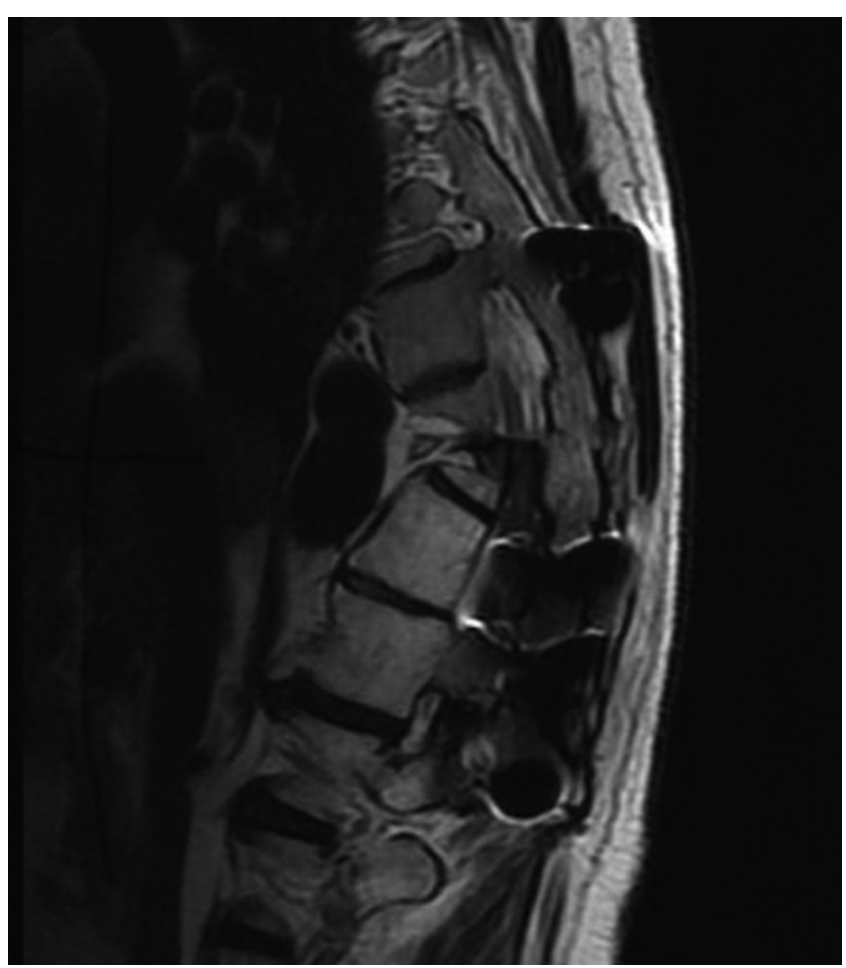

FIG. 2. Sagittal T2-weighted MR image obtained at the level of the arachnoid web seen on CT myelography, showing significant artifact from the Harrington rods.

\section{Postoperative Course}

Postoperative standing scoliosis radiographs revealed an improvement in the patient's scoliotic curve to approximately $14^{\circ}$ (Fig. 5). His postoperative recovery was uneventful, and by the 6-week follow-up, his preoperative symptoms had improved significantly with no burning in either leg, normal sensation in his left leg, and improved sensation in the right; his gait was continuing to improve as he was using a cane for support instead of a walker. At 3 months, his recovery had stabilized, and he had full strength in his lower extremities but remained hyper-reflexive and had mild persistent sensory changes. Unfortunately, some of the neuropathic pain persisted throughout his right lower extremity, although it was well managed with gabapentin. At the time of his 6-month follow-up, he reported a subjective increase in his lower-extremity spasticity, at a level close to where he was before surgery. Unfortunately, he also had a return of the hypersensitivity in his right lower extremity. On physical examination, his strength remained full at 5/5 in both lower extremities and he had persistent hyper-reflexia. Computed tomography myelography (Fig. 6) was repeated, demonstrating what appeared to be some reduced caliber of the spinal cord at the level of the web, probably representing atrophy. Otherwise, there was no evidence of CSF leakage or new stenosis. However, good decompression at the level of the resected pedicle at T-11 was evident on coronal views (Fig. 7).

\section{Discussion}

Here we describe a patient with congenital scoliosis due 

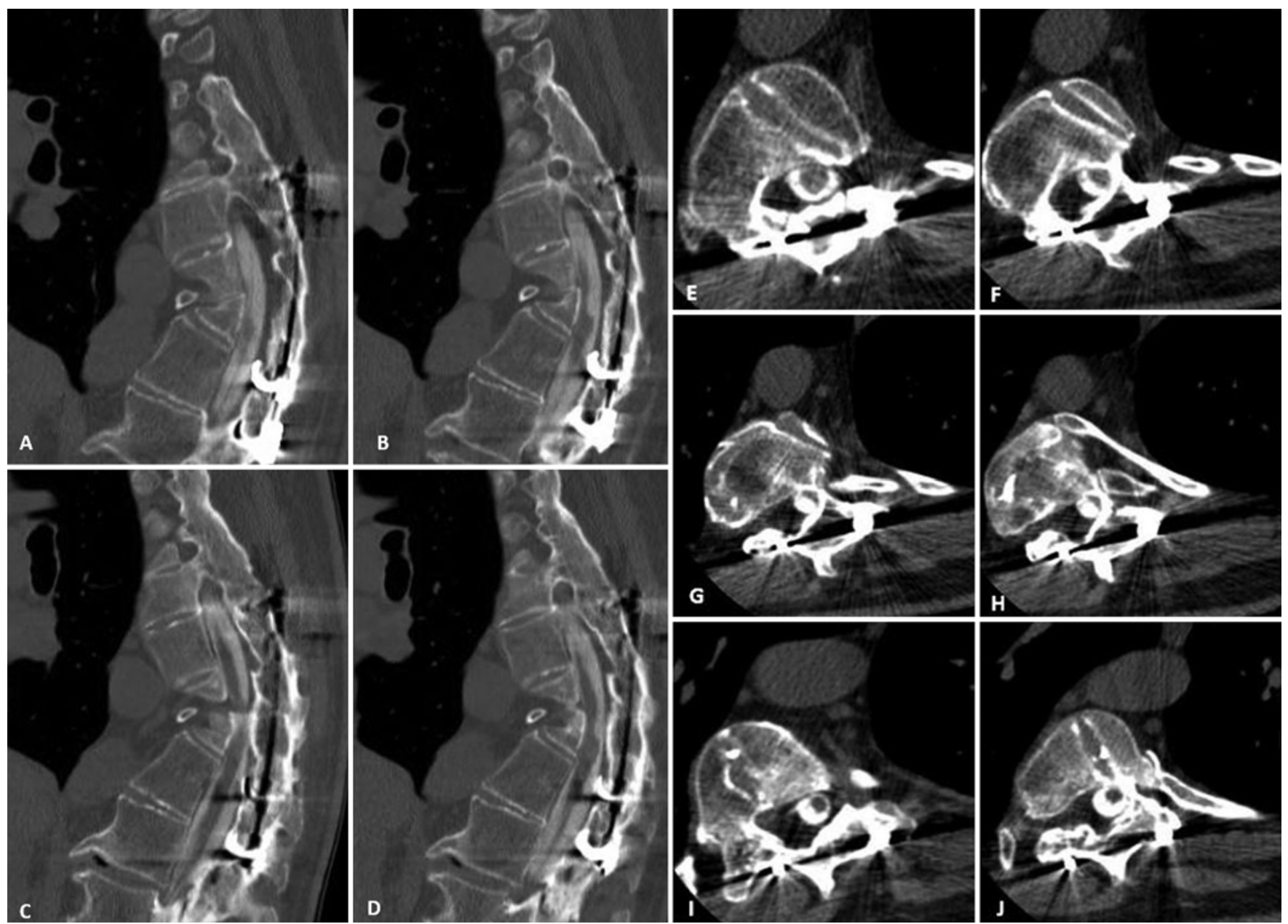

FIG. 3. Preoperative CT myelograms (A-D) demonstrating the "scalpel sign" at the T-11 level in the sagittal plane. Axial views $(\mathrm{E}-\mathrm{J})$ at the level of T-11 demonstrating significant cord compression.

to a hemivertebra requiring surgical stabilization in childhood, with subsequent presentation 41 years later as an adult with acute progressive myelopathy due to an arachnoid web. To our knowledge, this is the first published case of a thoracic hemivertebra with significant spinal canal stenosis secondary to a dorsal arachnoid web leading to myelopathy. Arachnoid webs are bands of arachnoid tissues that block normal CSF circulation through the spine. Their etiology is unknown, but they are hypothesized to develop either from diverticula of the septum posticum ${ }^{7}$ or, similar to an intracranial arachnoid cyst, from CSF production. ${ }^{5}$ Arachnoid webs typically present in the thoracic spine and have a "scalpel sign" on MRI or CT myelography, indicating spinal cord indentation from mass effect of the web approximating the outline of a surgical scalpel blade. ${ }^{8}$ Arachnoid webs are commonly associated with syringomyelia either above or below the lesion. ${ }^{5,9}$ Most operative reports mention surgeons identifying an adhesion between the pia and the thickened arachnoid causing the obstruction, $, 1,7,8$ which is consistent with our intraoperative findings. While in our case the T-11 hemivertebra at the apex of the scoliosis directed the surgeons to the area of concern, the web itself can only be seen on MRI or CT myelography indirectly by its mass effect on the spinal cord, potentially requiring better imaging techniques to localize the area of the web. ${ }^{3}$

Furthermore, it is not clear if arachnoid webs develop slowly and become symptomatic due to increased compression of the spinal cord or if they are an acute presentation. There is an association with syringomyelia in many cases; in our case, the patient was myelopathic without syringomyelia.

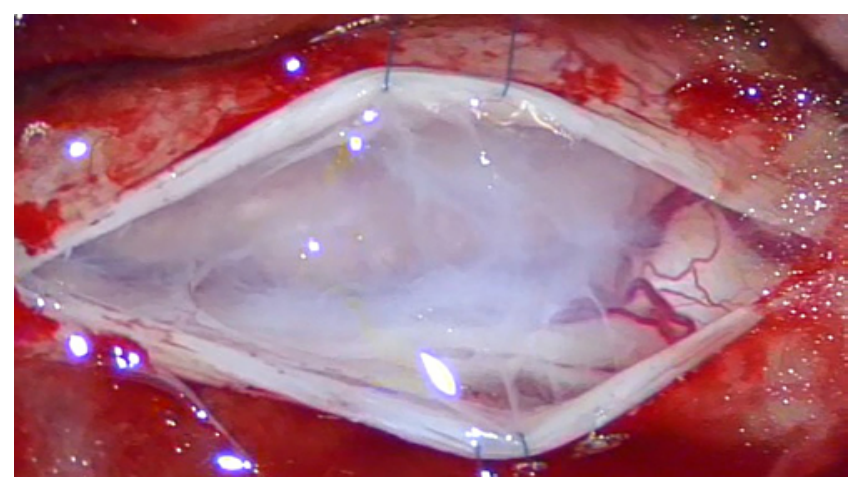

FIG. 4. Intraoperative view of the arachnoid web. Figure is available in color online only. 

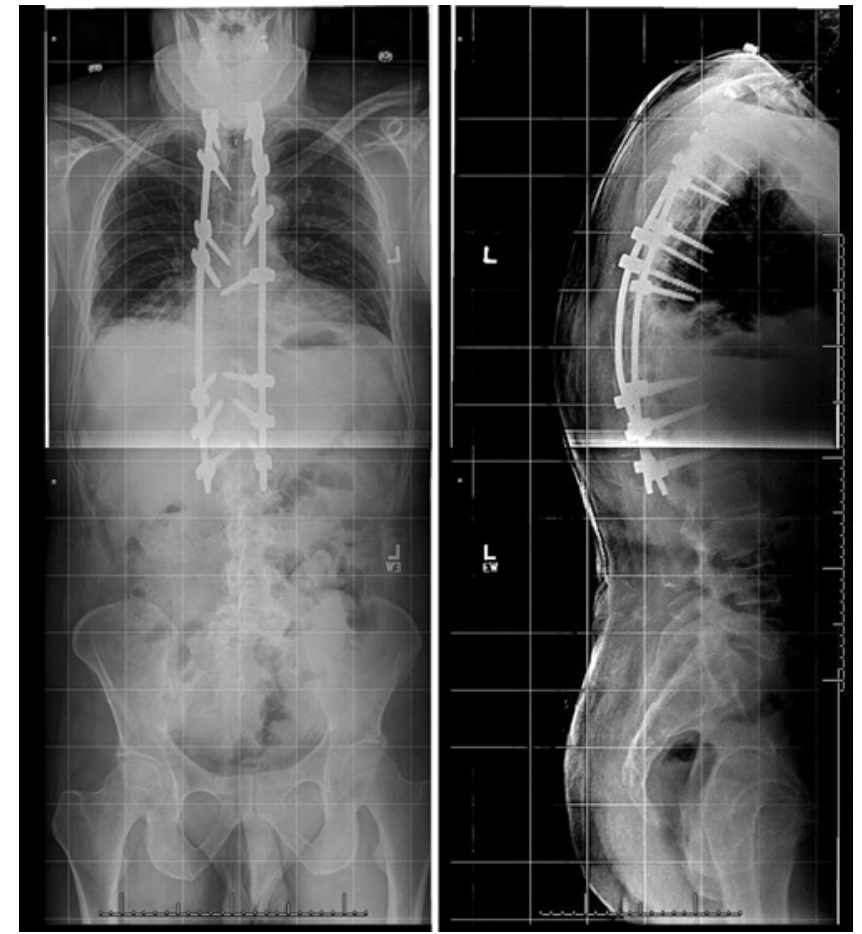

FIG. 5. Postoperative standing scoliosis radiographs demonstrating improvement in the scoliotic curve.

In our patient, there was no history of trauma that may have caused the development of an arachnoid web. One possibility is that there was a durotomy during his initial surgery, which led to scar formation and a predisposition to arachnoid web formation. It has been theorized ${ }^{8}$ that the dorsal arachnoid web leads to progressive changes in CSF dynamics and to syringomyelia. In our case, the progressive cord compression from an arachnoid web superimposed on a narrowed canal from scoliosis due to the hemivertebra probably led to the development of myelopathic symptoms.

In adult myelopathy, the literature on cervical myelopa-
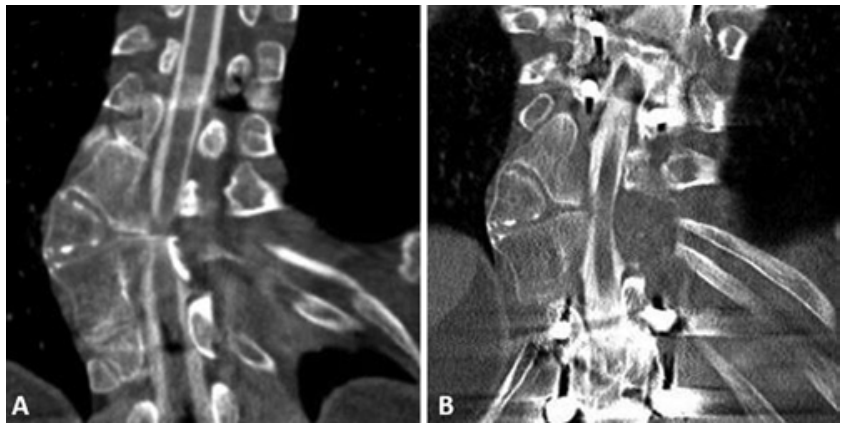

FIG. 7. Preoperative (A) and postoperative (B) coronal views demonstrating decompression after pedicle resection at the T-11 hemivertebra.

thy reports that improvements in symptoms after decompression occur in anywhere from $30 \%$ to $68 \%$ of patients, there is no change in $25 \%-30 \%$ of patients, and there is worsening in $5 \%-30 \%$ of patients. . $^{2,46}$ Studies have also suggested that patient age at the time of symptom onset has an effect on outcome as well. ${ }^{6}$ In our case, it is not clear if the patient's problem was associated more with the arachnoid web, the residual deformity, or, more likely, a combination of the two. Given his progressive deterioration before surgery, the goal of intervention was to stop his neurological decline and to improve his neurological status. We are not aware of previous reports of neurological deterioration in the segments of an intact, stable fusion.

\section{Conclusions}

In patients with complex anatomy and symptoms unexplained by findings on MRI, CT myelography studies should be obtained. The presence of a scalpel sign should raise suspicion for an arachnoid web. Treatment of just the arachnoid web in our case would have placed the patient at risk for recurrent cord compression; therefore, deformity correction with pedicle resection at T-11 as well as intradural resection of the arachnoid web was performed with subsequent improvement in myelopathic symptoms.
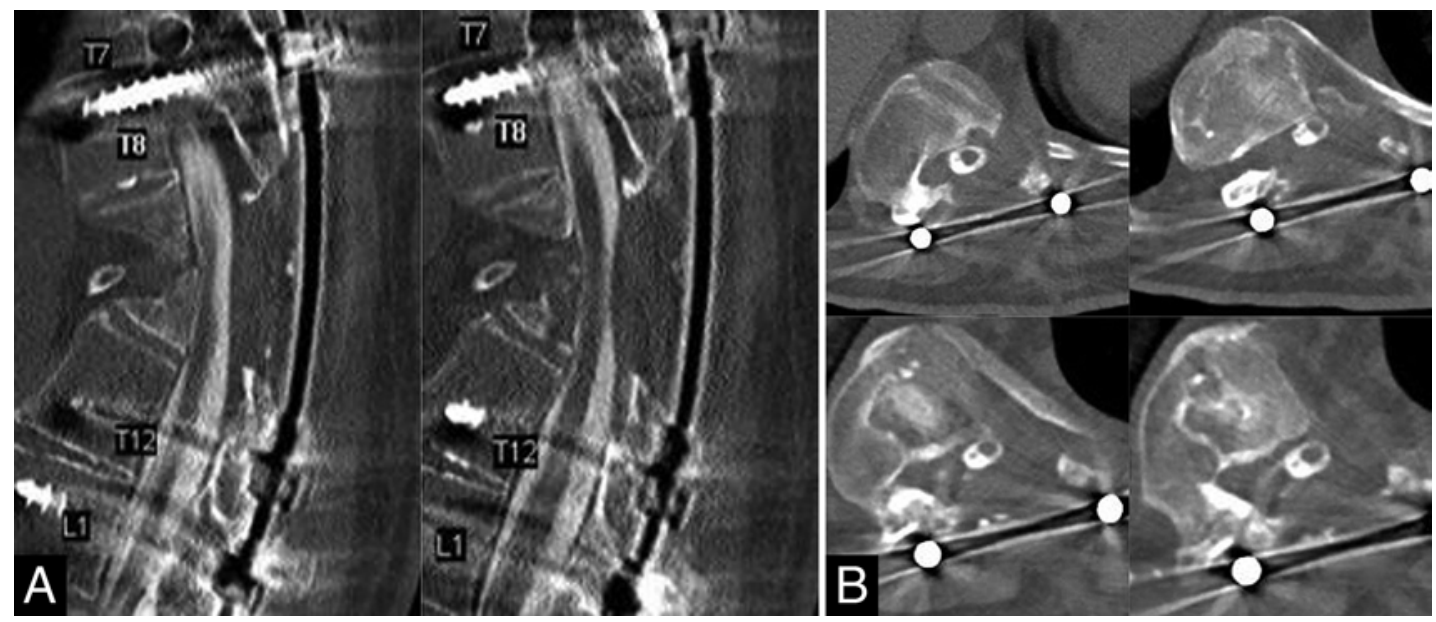

FIG. 6. Postoperative CT myelograms with atrophic cord at the level of the previous arachnoid web. There is no evidence of a CSF leak. A: Sagittal view from T-7 to L-1. B: Axial view at T10-11. 


\section{References}

1. Aiyer R, El-Sherif Y, Voutsinas L: Dorsal thoracic arachnoid web presenting as neuropathic pain: 'scalpel' sign found on MRI. Neuroradiol J 29:393-395, 2016

2. Bishara SN: The posterior operation in treatment of cervical spondylosis with myelopathy: a long-term follow-up study. J Neurol Neurosurg Psychiatry 34:393-398, 1971

3. Chang HS, Nagai A, Oya S, Matsui T: Dorsal spinal arachnoid web diagnosed with the quantitative measurement of cerebrospinal fluid flow on magnetic resonance imaging. J Neurosurg Spine 20:227-233, 2014

4. Fager CA: Results of adequate posterior decompression in the relief of spondylotic cervical myelopathy. J Neurosurg 38:684-692, 1973

5. Fortuna A, La Torre E, Ciappetta P: Arachnoid diverticula: a unitary approach to spinal cysts communicating with the subarachnoid space. Acta Neurochir (Wien) 39:259-268, 1977

6. Handa Y, Kubota T, Ishii H, Sato K, Tsuchida A, Arai Y: Evaluation of prognostic factors and clinical outcome in elderly patients in whom expansive laminoplasty is performed for cervical myelopathy due to multisegmental spondylotic canal stenosis. A retrospective comparison with younger patients. J Neurosurg 96 (2 Suppl):173-179, 2002

7. Paramore CG: Dorsal arachnoid web with spinal cord compression: variant of an arachnoid cyst? Report of two cases. J Neurosurg 93 (2 Suppl):287-290, 2000

8. Reardon MA, Raghavan P, Carpenter-Bailey K, Mukherjee
S, Smith JS, Matsumoto JA, et al: Dorsal thoracic arachnoid web and the "scalpel sign": a distinct clinical-radiologic entity. AJNR Am J Neuroradiol 34:1104-1110, 2013

9. Sayal PP, Zafar A, Carroll TA: Syringomyelia secondary to "occult" dorsal arachnoid webs: report of two cases with review of literature. J Craniovertebr Junction Spine 7:101104, 2016

\section{Disclosures}

The authors report no conflict of interest concerning the materials or methods used in this study or the findings specified in this paper.

\section{Author Contributions}

Conception and design: Hubbard, Hunt, Polly. Acquisition of data: Hubbard, Hunt, Polly. Analysis and interpretation of data: Hubbard. Drafting the article: Hubbard. Critically revising the article: Hunt, Jones, Polly. Reviewed submitted version of manuscript: all authors. Approved the final version of the manuscript on behalf of all authors: Hubbard. Administrative/technical/material support: Hubbard.

\section{Correspondence}

Molly Hubbard, Department of Neurosurgery, University of Minnesota, 420 Delaware St. SE, Mayo MMC-96, Minneapolis, MN 55455.email: hubba166@umn.edu. 